\title{
Estructura de la comunidad y asociación de las aves acuáticas con la heterogeneidad espacial del complejo lagunar Bahía Magdalena-Almejas, Baja California Sur, México
}

\author{
Bulmara Zárate-Ovando ${ }^{1}$, Eduardo Palacios ${ }^{2} \&$ Héctor Reyes-Bonilla ${ }^{3}$ \\ 1 Centro de Investigaciones Biológicas del Noroeste, Apdo. Postal 128, Mar Bermejo \# 195, Playa Palo de Santa Rita, \\ La Paz, Baja California Sur, 23090, México; bzarate04@cibnor.mx \\ 2 Centro de Investigación Científica y de Educación Superior de Ensenada, B.C. (CICESE) Unidad La Paz. Miraflores \\ 334, Fracc. Bellavista, La Paz, Baja California Sur, 23050, México; epalacio@cicese.mx \\ 3 Departamento de Biología Marina, Universidad Autónoma de Baja California Sur, Apdo. Postal 19-B, La Paz, Baja \\ California Sur, C.P. 23080, México; hreyes@uabcs.mx
}

\author{
Recibido 18-VIII-2006. Corregido 29-IV-2007. Aceptado 10-VIII-2007.
}

\begin{abstract}
Community structure and association of waterbirds with spatial heterogeneity in the Bahía Magdalena-Almejas wetland complex, Baja California Sur, Mexico. To test the hypothesis that spatial heterogeneity determines waterbird diversity in a coastal wetland, we compared waterbird density, diversity, and species composition among various habitats and landscapes units in Bahía Magdalena-Almejas, Baja California Sur, Mexico. Based on diversity patterns and bird distribution, we assessed the effect of coastal development on the waterbird community structure. To gather information on habitat features and waterbird populations we conducted waterbird censuses in 329 habitat segments along the internal coastline of the study area, from February 2002 to February 2003. We performed non-parametric analyses to test the null hypothesis of no diference on waterbird density, and diversity among landscape units and seasons. The species composition was evaluated using ordination techniques. Waterbird density was higher in winter and autumn in the three lagoons of the study area, particularly in the insular coast, sandy beach and dunes; it was higher in the few segments of antropic sustrate. Diversity was significantly higher in mangrove habitat along the peninsular coast, and in mangrove-dunes association on the insular coast. Although modification of coastal natural habitat and boat transit disturbance had no influence on waterbird density or diversity, the highest richness of waterbirds occurred in well preserved areas. Species composition analysis showed differences between Santo Domingo Channel and Magdalena Bay. Pelagic and mangrove habitat had a species composition difference higher than those associations in other habitats. Such differences in species assemblages by habitat and landscape units suggest that communities are structured according to the range of available natural resources in structurally complex habitats, and that dominant piscivorous waterbird species were favored in this community. Rev. Biol. Trop. 56 (1): 371-389. Epub 2008 March 31.
\end{abstract}

Key words: Bahía Magdalena-Almejas, Baja California Sur, coastal zones, community structure, density, disturbance, heterogeneity, waterbirds.

La distribución de las aves no es uniforme en las zonas costeras, sino que se relaciona con la disponibilidad y concentración temporal del alimento (Weller 1999), que a su vez se rige de procesos y factores ambientales (Butler et al. 1997, Weller 1999, Roshier et al 2001, Becker y Bissinger 2003, Jaquement et al. 2004). El hábitat, a nivel de paisaje o microhabitat, es uno de los factores ambientales que define el patrón de diversidad y el tipo de comunidad, porque limita la presencia de determinados grupos de aves (Weller 1999, Hattori y Mae 2001). De tal modo que en la línea de costa, aquellos humedales con diferentes tipos de 
habitat tendrán menor o mayor diversidad, y diferente composición.

En los humedales de la península de Baja California, gran parte del conocimiento de las aves acuáticas se ha generado de evaluaciones sistemáticas anuales de la abundancia total de aves o abundancia específica a nivel de laguna o localidad (Amador 1985, Guzmán et al 1994, Carmona 1995, Carmona y Danemann 1998, Fernández et al. 1998, Carmona y Carmona 2000, Castillo-Guerrero y Carmona 2001). Recientemente, en el complejo lagunar Bahía Magdalena-Almejas (CLBM-A), se determinó la distribución y abundancia de las aves acuáticas, para las tres lagunas que conforman este humedal (Zárate-Ovando et al. 2006). Aunque esta información es muy útil para actualizar listados de especies (Grinnell 1928, Nelson 1920, Wilbur 1987, Massey y Palacios 1994, Erickson y Howell 2001, Howell 2001, Howell et al. 2001), comparar cambios temporales y espaciales de la abundancia poblacional en sitios particulares (Guzmán et al. 1994, Palacios et al. 1994, Carmona 1995, Page et al. 1997, Carmona y Danemann 1998, Escofet et al. 1988, Fernández et al. 1998, Carmona y Carmona 2000, Castillo-Guerrero y Carmona 2001, Amador et al. 2006), o riqueza relativa (Amador 1985) de los humedales, ofrece poca información sobre la estructura de la comunidad y su relación con factores ambientales, como la complejidad y heterogeneidad de hábitat, disturbio, clima, o con procesos ligados a la productividad biológica local o de mesoescala, y eventos históricos.

Por lo anterior, este trabajo plantea, que por medio de un inventario de aves acuáticas del CLBM-A de un estudio extensivo que abarcó la costa interna de las lagunas de este humedal, analizar la comunidad con base en la densidad, diversidad y composición de aves acuáticas a diferentes escalas espaciales y las asociadas a los cambios temporales, para evaluar el efecto de 1) las unidades de paisaje tales como lagunas, zonas y hábitats, y el estado de conservación del litoral del CLBM-A sobre los atributos de la comunidad aviar. Las predicciones fueron que en otoño e invierno se observaría: 1) mayor densidad y diversidad de aves en lagunas o costas (insular y peninsular) que tuvieran mayor número de habitats o habitats con estructura compleja; y 2) que las costas con disturbio presentarían diversidad menor que la costa sin disturbio. Los resultados que se obtengan constituirán la línea base de indicadores que coadyuven a futuro en la implementación de planes de manejo para la conservación de las aves acuáticas y de la biodiversidad del CLBM-A.

\section{MATERIALES Y MÉTODOS}

Área de estudio: el estudio presente se llevó a cabo en el complejo lagunar Bahía Magdalena-Almejas $\left(24^{\circ} 30^{\prime}-25^{\circ} 80^{\prime} \quad \mathrm{N}\right.$ y $\left.111^{\circ} 50^{\prime}-112^{\circ} 20^{\prime} \mathrm{W}\right)$, que se localiza en la costa del Pacífico de la península de Baja California, es el sistema lagunar más grande de la región (1 $875 \mathrm{~km}^{2}$; Saad y Palacios 2004). Consta de tres lagunas: el Canal Santo Domingo (276 $\left.\mathrm{km}^{2}\right)$, Bahía Magdalena $\left(721 \mathrm{~km}^{2}\right)$ y Bahía Almejas $\left(383 \mathrm{~km}^{2}\right)$. La laguna mas profunda es Bahía Magdalena (40 m en la boca). Dos barras arenosas y tres islas limitan a este complejo lagunar con el Océano Pacífico; Las Ánimas es la barra más norteña e Isla Creciente la más sureña, y entre ellas se localizan las islas Magdalena y Margarita. El complejo lagunar Bahía Magdalena-Almejas es productivo por la alta concentración de nutrientes y material particulado que provienen del sistema terrestre $\mathrm{y}$ de surgencias costeras (Zaytsev et al. 2003) y frentes oceánicos (Malakoff 2004). En el Canal Santo Domingo se encuentran grandes extensiones de bosque de manglar sobre planicies de inundación, entre dunas, o asociado con parches de marismas (Saad y Palacios 2004). En las tres lagunas, la comunidad de pastos marinos (Zostera marina y Phillospadix sp.) concentra alimento para las aves acuáticas. Asimismo, parches de costa rocosa, playa arenosa, dunas activas e inactivas y acantilados, conforman la heterogeneidad del litoral peninsular e insular (Saad y Palacios 2004). Y las aves utilizan estos habitats como áreas de 
alimentación, anidacion, invernación, refugio, muda o descanso durante la migración hacia latitudes sureñas o norteñas.

El estado de conservación del litoral se dividió en tres categorías: 1) Sin disturbio aparente (Sd), ó aquellos segmentos de litoral sin modificación a la condición natural del paisaje o segmentos del ambiente pelágico con tráfico ocasional de embarcaciones; 2) Con disturbio (d), ó segmentos de costa con la presencia de comunidades humanas semi-permanentes o permanentes como los campamentos pesqueros y acuícolas, y los poblados de menos de 100 habitantes (en total se identificaron 12 asentamientos humanos con estas características), estos sitios tienen trafico semi-continuo de embarcaciones derivado de las actividades que se desarrollan en cada campamento o localidad; y 3) Con disturbio y modificación mayor (d c-m) a la condición natural, en esta categoría se integraron los segmentos con modificaciones ocasionada por actividades humanas de los poblados de más de 100 habitantes. Los poblados que destacan en el CLBM-A con estas características son: Puerto López Mateos, Puerto San Carlos, Puerto
Alcatraz, Puerto Cortés y Puerto Chale, donde se observa la presencia de muelles y otras edificaciones habitacionales o industriales (termoeléctrica, conservera).

Sistema de estudio: se realizaron 12 censos diurnos en el complejo lagunar Bahía Magdalena-Almejas, de febrero 2002 a febrero 2003 (ver Zárate-Ovando 2007). A lo largo de la costa interna de las tres lagunas, cuatro observadores expertos identificaron y contaron las aves acuáticas en 329 segmentos de habitat que recorrieron simultáneamente en dos embarcaciones. Los censos se realizaron durante cuatro días de primavera, dos de verano, tres de otoño, y tres de invierno.

Heterogeneidad espacial: se identificaron doce tipos de habitats, once de la zona intermareal que se diferenciaron por sus características fisonómicas incluyendo la cobertura vegetal y sustratos; y el habitat pelágico, que se limitó a dos transectos longitudinales en el cuerpo de agua de Bahía Magdalena y las bocas de las lagunas (Cuadro 1).

CUADRO 1

Longitud total $(\mathrm{km})$ de hábitat recorrida por laguna en el área de estudio

TABLE 1

Total length of habitat censused by lagoon at the study area $(N=328)$

Canal Santo Domingo

Manglar
Dunas
Playa arenosa
Costa rocosa
Sustrato antrópico
Manglar-dunas
Planicie inundación
Vegetación terrestre
Manglar-marismas
Islotes-manglar
Pelagico
Acantilados
Totales

1609

183

13

22

2

57

9

0

16

131

0

0

2040
Bahía Magdalena

255

90

209

89

$$
6
$$

0

0

0

4

0

304

24

983
Bahía Almejas 255

21

118

0

0

42

0

27

2

0

0

0

466 
Se diferenciaron cuatro tipos de manglar con base en el tipo de vegetación o sustrato adyacente: 1) Asociado a marismas, 2) asociado a planicies de inundación, 3) entre dunas, e 4) islotes de mangle. La mayoría de los islotes se encuentran a lo largo del Canal Santo Domingo. Otros habitats fueron: la planicie de inundación, playa arenosa, costa rocosa, dunas, parches de vegetación terrestre, y los acantilados que en la costa interna del CLBM-A se localizan en el noroeste de isla Santa Margarita principalmente. En total se recorrió una longitud mayor de manglar, dunas y playa arenosa (Cuadro 1) que de los otros habitats porque la cobertura de estos es mayor en el CLBM-A.

El área del CLBM-A, de las lagunas, y la longitud de la línea de costa interna de las lagunas y segmentos de hábitat se cuantificaron a partir de una base espacial corregida del área de estudio (Zárate-Ovando et al. 2006). El Canal Santo Domingo fue la laguna más somera y de menor área, pero tiene la línea de costa interna tres veces mayor $(1368 \mathrm{~km})$ que la de Bahía Magdalena (463 km), y aproximadamente cuatro veces mayor que la de Bahía Almejas (299 km).

Monitoreo de la comunidad de aves: para los censos se emplearon guías de campo (Sibley 2001, National Geographic Society 2002), binoculares (8 y 10x) y telescopios (Zoom 25-60x). Se realizaron conteos directos en parvadas menores a 300 individuos, en mayores la abundancia se estimó (Kasprzyk y Harrington 1989). Se incluyeron otras especies que se asocian al ambiente acuático, tales como el martín pescador norteño Ceryle alcyon, el gavilán pescador Pandion haliaetus, el águila cabeza blanca Haliaeetus leucocephalus, el halcón peregrino Falco peregrinus y el chipe amarillo Dendroica petechia. Cabe mencionar que además de registrar en cada segmento las aves posadas sobre los sustratos o coberturas vegetales, se sumaron los individuos de las especies que volaban en la superficie de los mismos (solo en el caso de las tijeretas, se tomaron en cuenta los individuos que volaban entre la superficie y $30 \mathrm{~m}$ de altura); o las que nadaban en el agua circundante de los primeros 200 m de la línea de costa.

Análisis de datos: con los datos de abundancia y longitud de los segmentos se calculó la densidad, que es el número de individuos por cada $60 \mathrm{~m}$ de segmento, esta longitud fue la mínima censada. Debido a que se recorrieron diferentes longitudes de segmentos de habitat (0.060-219 km; $10.59 \pm 23.00 \mathrm{~km}$; Cuadro 2), la densidad de las aves se transformó a $\log _{\mathrm{e}}$ (densidad de aves +1) para estabilizar la variancia (Clarke y Warwick, 2001) por la presencia de valores extremos de densidad de algunas especies en sitios particulares.

La comunidad se describió por métodos univariados o estimadores tradicionales de biodiversidad que se basaron en la densidad de las especies tales como el de diversidad de Shannon H', este índice se basó en el log base e y se calculó con base en los valores de densidad de las especies de cada segmento.

La variabilidad espacial y temporal de la densidad, diversidad y composición se cuantificó a través de un diseño jerarquizado de datos, considerando como bloques a los segmentos o repeticiones incluidos en: 1) las estaciones del año (primavera, verano, otoño e invierno); 2) lagunas (Canal Santo Domingo, Bahía Magdalena, Bahía Almejas); 3) zonas (costa insular, costa peninsular, pelágico); 4) hábitats (acantilados, pelágico, dunas, islotes de manglar, manglar, manglar-marismas, manglardunas, planicie de inundación, playa arenosa, costa rocosa, vegetación terrestre y sustrato antrópico; 5) y según el estado de conservación aparente del litoral del CLBM-A según las categorías antes descritas a) Sin disturbio sd, b) Con disturbio d, y c). disturbio costamodificada dc-m.

La prueba de hipótesis de no diferencia de densidad de aves entre las estaciones del año, lagunas, zonas, habitats o en el litoral con diferente estado de conservación se realizó por medio de la prueba Kruskal-Wallis y con la prueba Chi-cuadrado de medianas. Las pruebas a posteriori se realizaron por medio de comparaciones múltiples. 
CUADRO 2

Longitud de línea de costa censada por segmentos $(N=328)$, en las temporadas y unidades de paisaje

TABLE 2

Length of the line coast censused by segments $(N=328)$, in the seasons and landscape units

Longitud de los segmentos (km)

$\mathrm{N} \quad$ Promedio $\pm \begin{gathered}\text { Desv. Mín. Máx. } \\ \text { Est. }\end{gathered}$ $\begin{array}{cc}\text { Línea } & \text { Riqueza } \\ \text { de costa censada } & \text { Acumulada }\end{array}$

Total $(\mathrm{km}) \quad \mathrm{S}$

\section{TEMPORADAS}

\begin{tabular}{|c|c|c|c|c|c|c|c|c|}
\hline Primavera & 122 & 7.21 & \pm & 11.52 & 0.38 & 108 & 879 & 56 \\
\hline Verano & 72 & 13.35 & \pm & 34.98 & 0.14 & 219 & 962 & 53 \\
\hline Otoño & 68 & 14.49 & \pm & 22.82 & 0.21 & 141 & 985 & 70 \\
\hline Invierno & 66 & 9.80 & \pm & 22.26 & 0.06 & 125 & 647 & 58 \\
\hline \multicolumn{9}{|l|}{ LAGUNAS } \\
\hline Canal Santo Domingo & 136 & 14.97 & \pm & 33.54 & 0.06 & 219 & 2036 & 73 \\
\hline Bahía Magdalena & 140 & 7.01 & \pm & 9.41 & 0.14 & 55 & 981 & 66 \\
\hline Bahía Almejas & 52 & 8.77 & \pm & 9.20 & 0.26 & 32 & 456 & 52 \\
\hline \multicolumn{9}{|l|}{ ZONAS } \\
\hline Costa peninsular & 160 & 14.19 & \pm & 29.36 & 0.14 & 219 & 2270 & 75 \\
\hline Costa insular & 124 & 7.22 & \pm & 15.64 & 0.06 & 125 & 896 & 67 \\
\hline Pelágico & 44 & 6.98 & \pm & 6.42 & 0.29 & 31 & 307 & 19 \\
\hline \multicolumn{9}{|l|}{ HABITATS } \\
\hline Acantilados & 8 & 2.33 & \pm & 1.02 & 1.51 & 5 & 19 & 13 \\
\hline Pelágico & 44 & 6.98 & \pm & 6.42 & 0.29 & 31 & 307 & 19 \\
\hline Dunas & 34 & 8.68 & \pm & 21.07 & 0.12 & 125 & 295 & 60 \\
\hline Islote-Manglar & 7 & 18.25 & \pm & 42.60 & 0.06 & 115 & 128 & 20 \\
\hline Manglar & 134 & 15.83 & \pm & 31.34 & 0.27 & 219 & 2122 & 71 \\
\hline Manglar-marismas & 10 & 2.25 & \pm & 1.58 & 0.07 & 4 & 22 & 32 \\
\hline Manglar-dunas & 12 & 8.05 & \pm & 9.73 & 0.98 & 32 & 97 & 51 \\
\hline Planicie inundación & 5 & 1.71 & \pm & 1.32 & 0.86 & 4 & 9 & 26 \\
\hline Playa arenosa & 51 & 6.63 & \pm & 9.55 & 0.21 & 46 & 338 & 53 \\
\hline Costa rocosa & 15 & 6.85 & \pm & 8.86 & 0.28 & 30 & 103 & 33 \\
\hline Vegetación terrestre & 3 & 9.07 & \pm & 14.41 & 0.26 & 26 & 27 & 14 \\
\hline Sustrato antrópico & 5 & 1.29 & \pm & 1.48 & 0.14 & 4 & 6 & 24 \\
\hline \multicolumn{9}{|c|}{ CONSERVACIÓN DEL LITORAL } \\
\hline Sin disturbio & 251 & 9.79 & \pm & 19.97 & 0.06 & 219 & 2458 & 76 \\
\hline Disturbio & 62 & 14.56 & \pm & 33.85 & 0.14 & 203 & 903 & 64 \\
\hline Disturbio costa modificada & 15 & 7.45 & \pm & 12.46 & 0.21 & 46 & 112 & 45 \\
\hline
\end{tabular}


Los valores de diversidad y equidad de los habitats calculados con base en la densidad de las especies de cada segmento, se usaron para comparar la estructura comunitaria entre temporadas y unidades de paisaje. La hipótesis de no diferencia en la diversidad y equidad de aves acuáticas entre estas se probó con las pruebas de estadística no paramétricas antes mencionadas. De los 329 segmentos se excluyó un segmento por tener el valor más extremo del índice de abundancia y solo una especie.

El grado de similitud de la composición específica entre las temporadas y unidades de paisaje (lagunas, zonas, habitats y su condición), se estimaron usando el índice de similitud de Bray Curtis, que considera en conjunto tanto a los datos de presencia- ausencia, y en este caso la densidad de las especies transformada a raíz 4ta (Clarke y Warwick 2001). A partir de esta matriz se realizó un análisis de ordenación por escalamiento multidimensional no métrico (NMDS o MDS; Clarke y Warwick 2001). Los modelos de ordenación se describieron con base en gráficos bi y tridimensionales MDS considerando el nivel estrés. El análisis de similitud ANOSIM de una vía para grupos de muestras se aplicó para comprobar las tendencias observadas de los modelos MDS y someter a prueba las hipótesis nulas Ho: No existen diferencias entre las asociaciones entre grupos de muestras especificados a priori, en este caso entre temporadas, lagunas, zonas, habitats y estado de conservación del litoral.

\section{RESULTADOS}

\section{Distribución espacial y temporal de la densidad de aves acuáticas}

La densidad de las aves acuáticas varió a lo largo del año, tanto en tiempo como en espacio (Cuadro 4). En general $(\mathrm{N}=328$ segmentos), la densidad fluctuó entre (0.011-2 189 ind ${ }^{*} 60 \mathrm{~m}^{-1}$ ) (Cuadro 4). Hubo variación temporal significativa de la densidad con base en los ámbitos y medianas $\left(\mathrm{H}_{3 \mathrm{~g} .1 .}=24.21, \mathrm{p}<0.05\right.$; Chi cuadrada $_{\text {3g.l. }}=24.24, \mathrm{p}<0.05$; Cuadro 4).
$\mathrm{Y}$ en las unidades de paisaje como las zonas $\left(\mathrm{H}_{2 \mathrm{~g} .1 .}=48.87, \mathrm{p}<0.05\right.$; Chi cuadrada ${ }_{3 \mathrm{~g} .1 .}=35.13$, $\mathrm{p}<0.05)$, lagunas $\mathrm{y}$ habitats $\left(\mathrm{H}_{11 \mathrm{~g} .1 .}=72.11\right.$, $\mathrm{p}<0.05$; Chi cuadrada ${ }_{11 \text { g.l. }}=55.63, \mathrm{p}<0.05$ ). Los ámbitos y las medianas de densidad aumentaron significativamente de primavera a invierno. Las pruebas a posteriori demostraron que la densidad de aves en verano e invierno fue significativamente mayor que en primavera $(p<0.05)$ en todo el complejo lagunar. Entre lagunas hubo diferencias de densidad con base en los ámbitos $\left(\mathrm{H}_{2 \mathrm{~g} .1}=6.11, \mathrm{p}<0.05\right)$, pero con base en las medianas no hubo diferencias significativas entre ellas (n.s.). Canal Santo Domingo fue la laguna con densidad diferente a las otras lagunas $(\mathrm{p}<0.05)$, y algunos segmentos de las tres lagunas tuvieron densidad muy alta. Entre zonas, las costas peninsular e insular concentraron significativamente una densidad de aves acuáticas mayor que la zona pelágica $(p<0.05)$. La costa peninsular tuvo una densidad de aves mayor que la costa insular, aunque existieron algunos segmentos de costa insular que concentraron densidades mayores, especialmente en Isla Patos y en colonias de anidación. Entre habitats (Cuadro 3 y 4), la densidad del ambiente pelágico fue significativamente menor que la de siete habitats $(\mathrm{p}<0.05)$; la densidad de aves del manglar fue menor que la de playa arenosa $(p<0.05)$ con base en los ámbitos de densidad, y con base en las medianas hubo mayor número de diferencias significativas $(p<0.05)$. Las diferentes categorías de conservación del litoral no tuvieron diferencias de densidad significativas $\left(\mathrm{H}_{2 \mathrm{~g} .1 .}=2.80\right.$, n.s.; Chi cuadrada $_{2 \mathrm{~g} \text {. } 1 \text {. }}=0.424$, n.s. $)$.

\section{Estructura de la comunidad}

Riqueza: en este trabajo se identificaron 80 especies. El cormorán orejudo Ph. auritus fue la especie con densidad acumulada mayor (30\% del total) y la segunda especie con mayor presencia en el litoral del CLBM-A (77 \% de los 329 segmentos censados). El pelicano café $P$. occidentalis tuvo densidad acumulada menor (20\% del total) que el cormorán orejudo pero fue la especie que se observó con 
CUADRO 3

Estadísticos de prueba ANOSIM de las comparaciones pareadas entre habitats

TABLE 3

Statistics ANOSIM test of the pairwise comparison between habitats

Habitats
(Comparaciones pareadas)

Acantilados, Pelágico

Acantilados, Islote-manglar

Acantilados, manglar

Acantilados, manglar-marismas

Acantilados, manglar-dunas

Acantilados, Planicie inundación

Pelagico, Dunas

Pelagico, Islote-manglar

Pelagico, Manglar

Pelagico, Manglar-marismas

Pelagico, Manglar-dunas

Pelagico, Planicie inundación

Pelagico, playa arenosa

Pelagico, Costa rocosa

Pelagico, Vegetación terrestre

Pelagico, Sustrato antrópico

Dunas, Islote-manglar

Dunas, Manglar

Dunas, Manglar-marismas

Dunas, Costa rocosa

Islote-manglar, Manglar

Islote-manglar, Manglar-dunas

Islote-manglar, Playa arenosa

Islote-manglar, Costa rocosa

Manglar, Manglar-marismas

Manglar, Playa arenosa

Manglar, Costa rocosa

Manglar-marismas, Manglar-dunas

Manglar-marismas, Playa arenosa

Manglar-marismas, Costa rocosa

Manglar-dunas, Planicie inundación

Manglar-dunas, Costa rocosa

Manglar-dunas, Sustrato antrópico

Planicie inundación, Costa rocosa
Estadístico R

0.451

0.406

0.433

0.471

0.693

0.655

0.601

0.628

0.561

0.882

0.807

0.768

0.505

0.586

0.562

0.796

0.278

0.207

0.626

0.142

0.273

0.397

0.286

0.342

0.648

0.282

0.422

0.434

0.601

0.502

0.339

0.416

0.291

0.368
Nivel de significancia \%

$$
0.2
$$$$
0.1
$$$$
0.2
$$$$
0.4
$$$$
0.1
$$$$
0.3
$$$$
0.1
$$$$
0.1
$$$$
0.1
$$$$
0.1
$$$$
0.1
$$$$
0.1
$$$$
0.1
$$$$
0.1
$$$$
2.7
$$$$
0.1
$$$$
1.6
$$

$$
0.1
$$$$
0.1
$$$$
3.1
$$$$
2.4
$$$$
0.2
$$$$
0.4
$$$$
0.4
$$$$
0.1
$$$$
0.1
$$$$
0.1
$$$$
0.1
$$$$
0.1
$$$$
0.1
$$$$
2.3
$$$$
0.1
$$

3

1.4 $\mathrm{p}$

0.002

0.001

0.002

0.004

0.001

0.003

0.001

0.001

0.001

0.001

0.001

0.001

0.001

0.001

0.03

0.001

0.02

0.001

0.001

0.03

0.02

0.002

0.004

0.004

0.001

0.001

0.001

0.001

0.001

0.001

0.02

0.001

0.03

0.01
Permutaciones Numero de $\mathrm{R} s \geq \mathrm{R}_{\text {global }}$ posibles

Observadas

752538150

6435

muchas

43758

125970

1287

muchas

115775100

muchas

muchas

muchas

1906884

muchas

muchas

1035

1906884

22481940

muchas

muchas

muchas

muchas

50388

300674088

170544

muchas

muchas

muchas

646646

muchas

3268760

6188

17383860

6188

15504
1

$$
0
$$

1

3

0

2

0

0

0

0

0

0

0

0

26

0

15

0

0

30

23

1

3

3

0

0

0

0

0

0

22

0

29

13 


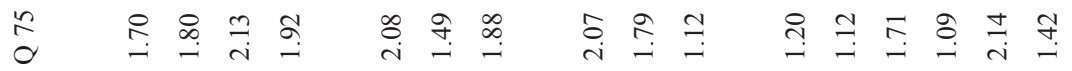

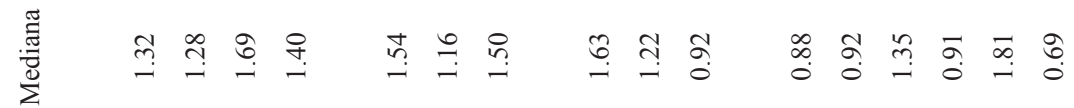

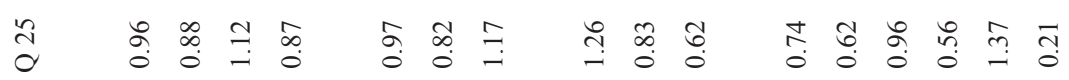

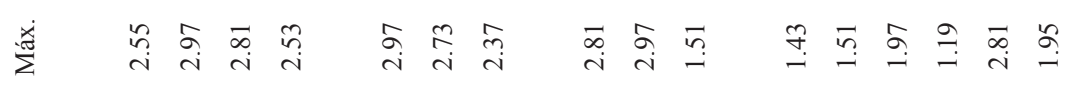

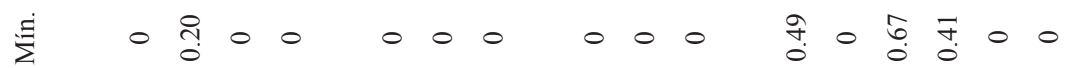

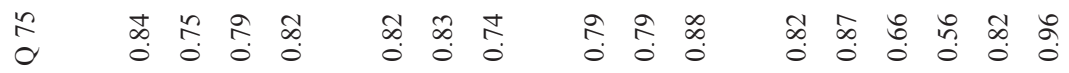

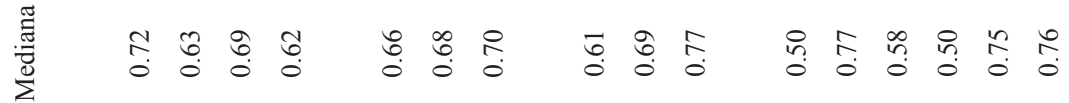
苞

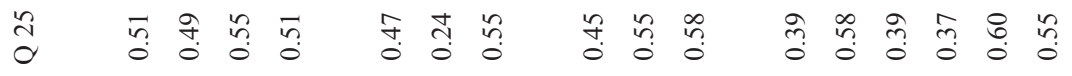

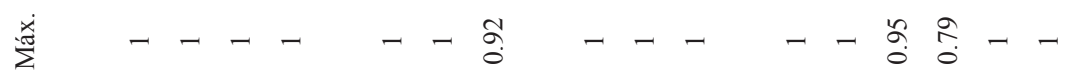

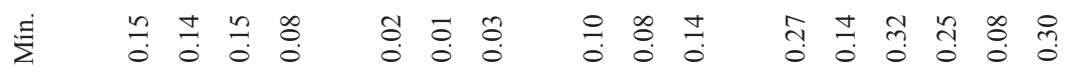

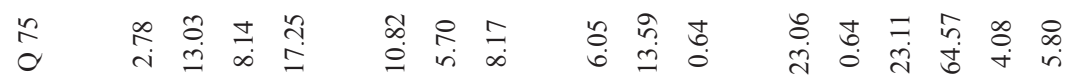

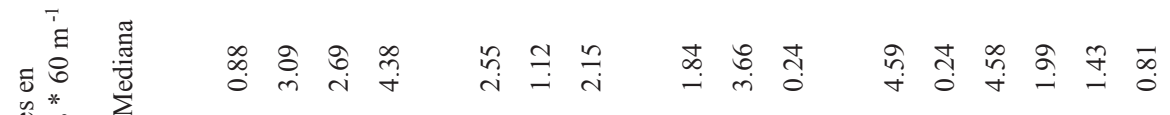
$\sum_{\substack{0 \\ 0}}^{*}$

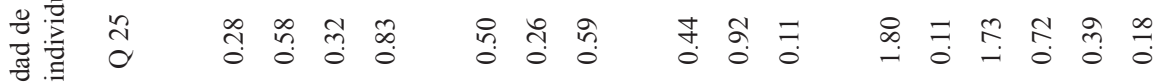
窇

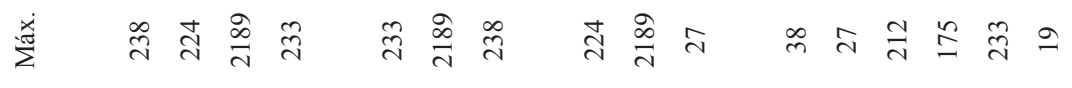

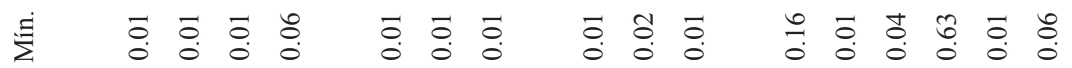

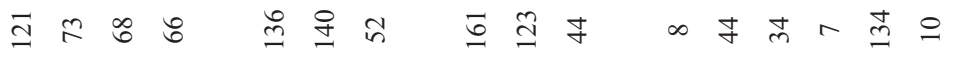

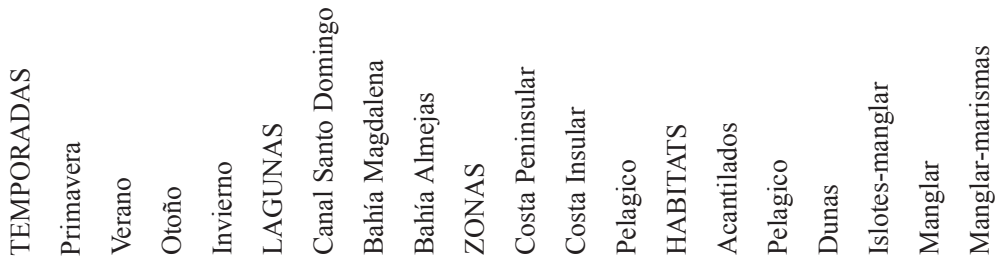




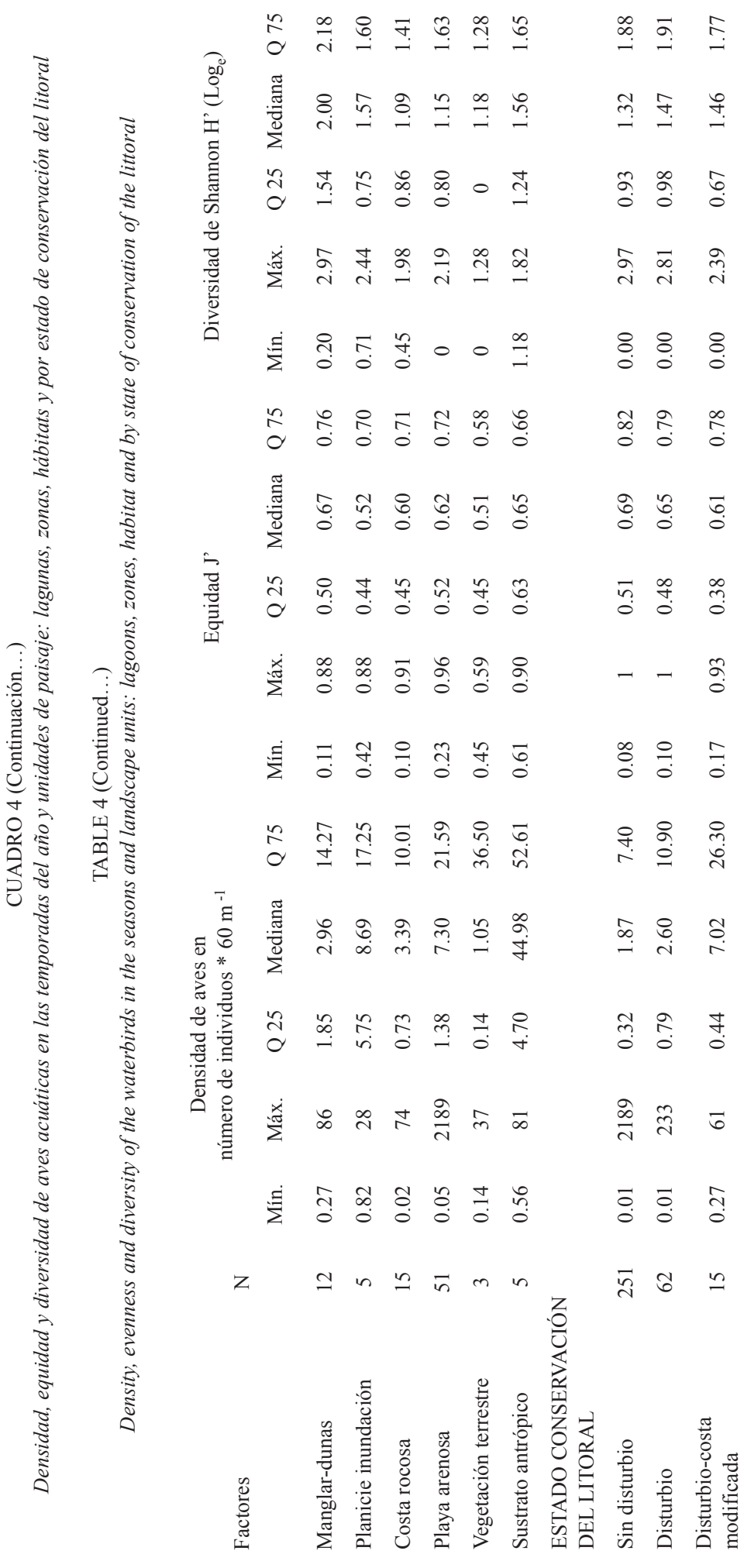


mayor frecuencia en el litoral del complejo lagunar ( $80 \%$ de los segmentos censados); y el cormorán de Brandt Ph. penicillatus aunque acumuló $17 \%$ de la densidad total, estuvo presente en tan solo $10 \%$ de los segmentos del litoral. En contraste, hubo 12 especies raras de las que solo se observaron diez individuos, de estas el bobo enmascarado Sula dactylatra, la cerceta canela Ana cyanoptera, el charrán negro Chlidonias niger y el halcón peregrino $F$. peregrino se observaron dos individuos, y del paíño negro Oceanodroma melania, el pato chillón Bucephala clangula, el pato tepalcate Oxyura jamaicensis y la avoceta americana Recurvirostra americana solo se observó un individuo.

En otoño hubo más especies (70 de las 80) que en el resto del año y en verano menos (53) (Cuadro 2). En las lagunas, Canal Santo Domingo fue donde se observaron más especies en total (73 especies) y menos en Bahía Almejas (52) (Cuadro 2). Entre zonas, la costa peninsular tuvo riqueza acumulada mayor (75) que la costa insular (67), en contraste en el ambiente pelágico se observaron tan solo 19 especies (Cuadro 2). Entre habitats, en manglar se observaron mas especies (71), seguido de dunas (60) y playa arenosa (53); mientras que en vegetación terrestre (14) y acantilados (13 especies) se observaron menor número de especies (Fig. 1). Finalmente, en la costa sin disturbio se observaron más especies (76 especies) que en las costas con disturbio (64) o con disturbio costa-modificada (45 especies).

En los doce habitats, el número de especies se incrementó irregularmente según la longitud de línea de costa censada (Fig. 1). En manglar se observó una riqueza de especies mayor que en dunas o playa arenosa, estos hábitats cubren la mayor parte del litoral del CLBM-A y en los que se censó mayor número de segmentos (Cuadro 1 y 2). Sin embargo, manglar-dunas con menor longitud de costa censada acumuló mayor número de especies que habitats como costa rocosa, islotes de manglar o pelágico donde se censó mayor longitud (Fig. 1).
Diversidad: Los valores de diversidad de aves por segmento variaron entre 0.00 a 2.97 bits (Cuadro 4). Hubo diferencias significativas de diversidad entre temporadas $\left(\mathrm{H}_{3 \mathrm{~g} . \mathrm{l}}=10.49\right.$, $\mathrm{p}<0.05$; Chi cuadrado ${ }_{3 \mathrm{~g} .1}=7.70, \mathrm{p}<0.05$ ) y unidades de paisaje, pero no entre grados de disturbio del litoral. Las pruebas Kruskall-Wallis y $a$ posteriori mostraron que: 1) Otoño fue la estación con diversidad significativamente mayor que primavera y verano $(\mathrm{p}<0.05)$, la segunda temporada fue la que tuvo diversidad menor (Cuadro 3 y 5). 2) En el caso de las lagunas $\left(\mathrm{H}_{2 \mathrm{~g} .1 .}=24.79, \mathrm{p}<0.05\right.$; Chi cuadrada ${ }_{2 \mathrm{~g} . \mathrm{l}}=19.97$, $\mathrm{p}<0.05$ ), Canal Santo Domingo y Bahía Almejas tuvieron diversidad significativamente más alta que Bahía Magdalena $(\mathrm{p}<0.05)$. 3) Entre las zonas, la diversidad de la costa peninsular fue significativamente mayor $\left(\mathrm{H}_{2 \mathrm{~g} .1 .}=60.45, \mathrm{p}<0.05\right.$; Chi cuadrada $\left.{ }_{2 \mathrm{~g} .1 .}=52.09, \mathrm{p}<0.05\right)$ que la de la costa insular $(\mathrm{p}<0.05)$ y el ambiente pelágico $(\mathrm{p}<0.05) .4)$. Entre los habitats $\left(\mathrm{H}_{11 \text { g. } .1}=107.6\right.$, $\mathrm{p}<0.05$; Chi cuadrado 11g.1. $\left._{102.08,} \mathrm{p}<0.05\right)$ el ambiente pelágico tuvo diversidad significativamente menor que dunas $(\mathrm{p}<0.05)$, manglar

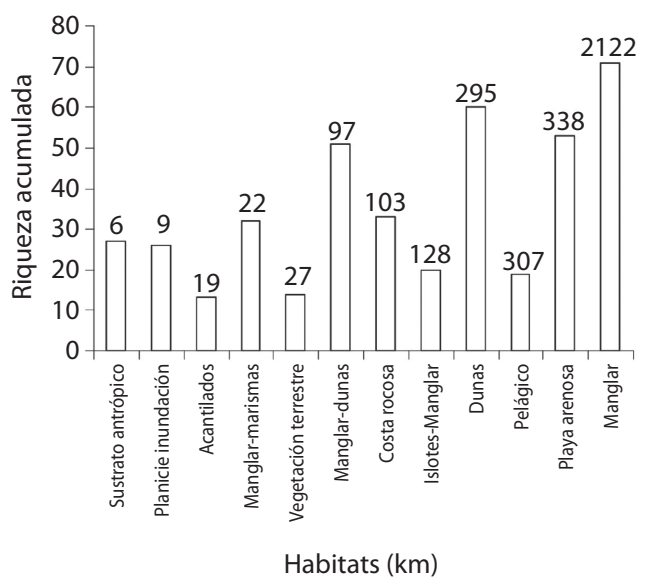

Fig. 1. Riqueza acumulada y longitud censada de cada habitat, los números sobre las barras indican los kilómetros recorridos de cada habitat.

Fig. 1. Accumulated richness and length censused in each habitat. Numbers on bars indicate kilometers surveyed in each habitat. 
$(\mathrm{p}<0.05)$ y manglar-dunas $(\mathrm{p}<0.05)$. En contraste, el manglar tuvo diversidad significativamente mayor que la de islotes de manglar y playa arenosa $(\mathrm{p}<0.05)$; y manglar-marismas tuvo diversidad significativamente menor que los islotes de manglar $(\mathrm{p}<0.05)$.

Equidad: La equidad de aves fue significativamente diferente entre temporadas con base en la diferencia de medianas únicamente (Chi cuadrada 3 g.l. $=6.27, \mathrm{p}<0.05$; Cuadro 4). $\mathrm{Y}$ de las unidades de paisaje solo las zonas $\left(\mathrm{H}_{2 \mathrm{~g} .1 .}=13.47, \mathrm{p}<0.05\right.$; Chi cuadrada 2 g.l. $=10.09$, $\mathrm{p}<0.05)$ y los habitats $\left(\mathrm{H}_{11 \mathrm{~g} .1 .}=40.21, \mathrm{p}<0.05\right.$; Chi cuadrada $\left._{11 \text { g.l. }}=39.56, \mathrm{p}<0.05\right)$ tuvieron diferencias de equidad de aves (Cuadro 4). Entre las zonas, la costa insular tuvo equidad significativamente menor que la costa peninsular $(\mathrm{p}<0.05)$ o el ambiente pelágico $(\mathrm{p}<0.05)$. Los habitats que tuvieron equidad mayor fueron manglar-marismas, ambiente pelágico, manglar y manglar-dunas. En contraste, los acantilados e islotes de manglar tuvieron equidad menor. La equidad del ambiente pelágico fue significativamente mayor que la de playa arenosa $(\mathrm{p}<0.05)$; así como la de manglar $v s$. dunas $(\mathrm{p}<0.05)$ y $v s$. playa arenosa $(\mathrm{p}<0.05) .5)$. En el litoral con diferente estado de conservación no hubo diferencias significativas de equidad.

Similitud: Los modelos de ordenación MDS de similitud relativa, temporal y espacial, mostraron agrupación de los segmentos de solo alguna temporada o unidad de paisaje con un stress (0.23-0.22) que indicó ausencia de tendencia. De las temporadas, invierno mostró dispersión mayor de los segmentos indicando que la composición en esta temporada fue diferente a las demás. Entre lagunas, los segmentos de Bahía Magdalena tendieron a agruparse; en contraste, los de Canal Santo Domingo tuvieron dispersión mayor que los de Bahía Almejas, es decir, la composición de Canal Santo Domingo mostró diferencia mayor que las otras lagunas. En las zonas, los segmentos del ambiente pelágico formaron una agrupación evidente, mientras que los de la costa insular tuvieron distancia menor a los segmentos del ambiente pelágico que los de la costa peninsular, ambas costas mostraron diferencia mayor en la composición en comparación con la del ambiente pelágico. En los habitats, los segmentos del ambiente pelágico mostraron agrupación mayor que indicó diferencia mayor de la composición en comparación con los demás habitats; los segmentos de manglar tuvieron agrupación mayor que los de las dunas y playa arenosa, los segmentos de estos dos habitats mostraron dispersión similar; en contraste, la composición de manglar-marismas se diferenció de la de los demás habitats porque mostró dispersión mayor de los segmentos. La composición de las costas con diferente estado de conservación mostró ausencia de tendencias de los segmentos a formar arreglos definidos y estos tuvieron dispersión amplia.

Los estadísticos de prueba ANOSIM revelaron que a pesar del alto traslape de los segmentos de las temporadas y unidades de paisaje hubo diferencias, temporal y espaciales, significativas de la composición de la comunidad de aves acuáticas. Entre estaciones del año hubo diferencias significativas $\left(\mathrm{R}_{\text {global }}=0.07, \mathrm{p}=0.001\right)$ en cinco de seis comparaciones pareadas entre: primavera $v s$. otoño $(\mathrm{R}=0.09, \mathrm{p}=0.001)$ e invierno $(\mathrm{R}=0.11, \mathrm{p}=0.001)$, y entre verano $(\mathrm{R}=0.11$, $\mathrm{p}=0.001) v s$. invierno $(\mathrm{R}=0.11, \mathrm{p}=0.001)$ y otoño $(\mathrm{R}=0.08, \mathrm{p}=0.001)$, y entre invierno $v s$. otoño $(\mathrm{R}=0.03$, $\mathrm{p}=0.004)$ por la presencia de especies veraneantes e invernantes.

Entre lagunas, los estadísticos de prueba ANOSIM comprobaron que la composición fue significativamente diferente entre ellas $\left(\mathrm{R}_{\text {global }}=0.11, \mathrm{p}=0.001\right)$ debido a que las asociaciones de Canal Santo Domingo vs. Bahía Magdalena tuvieron diferencia $(\mathrm{R}=0.19$, $\mathrm{p}=0.001$ ).

Las asociaciones de aves de las costas peninsular e insular y el ambiente pelágico fueron diferentes $\left(\mathrm{R}_{\text {global }}=0.20, \mathrm{p}=0.001\right)$. La composición del ambiente pelágico fue diferente a la de la costa insular $(\mathrm{R}=0.29, \mathrm{p}=0.001)$ y mostró diferencia mayor que la costa peninsu$\operatorname{lar}(\mathrm{R}=0.46, \mathrm{p}=0.001)$. Aunque las asociaciones entre costas también presentaron diferencias significativas entre sí $(\mathrm{R}=0.07, \mathrm{p}=0.001)$. 
La diferencia en la composición entre los hábitats descrita de los modelos MDS fueron comprobadas por la prueba ANOSIM, que reveló diferencias significativas $\left(\mathrm{R}_{\text {global }}=0.35\right.$, $\mathrm{p}=0.001$ ) comprobadas en 34 (Cuadro 3 ) de 66 comparaciones pareadas. La composición del ambiente pelágico fue significativamente diferente a la de los otros once habitats; la del manglar y manglar-marismas se diferenció de las de siete habitats respectivamente; la de acantilados e islotes de manglar tuvieron diferencias significativas con la composición de seis habitats respectivamente; la composición de dunas se diferenció de las de cinco habitats; la de planicie de inundación de la de cuatro habitats; y las asociaciones de sustrato antrópico y vegetación terrestre solo resultaron significativamente diferentes a la del ambiente pelágico.

Finalmente y consistente con el MDS de los segmentos de litoral con diferente estado de conservación, los estadísticos de prueba ANOSIM confirmaron que no hubo diferencias significativas de la composición entre ellos $\left(\mathrm{R}_{\text {global }}=0.02, \mathrm{p}=\right.$ n.s. $)$.

\section{DISCUSIÓN}

Nuestro estudio mostró que los cambios de la densidad, diversidad y composición de las especies de aves acuáticas fueron consecuencia del proceso migratorio y de la condición o recursos naturales de las lagunas, zonas y habitats, puesto que tuvieron efecto en la distribución de las especies y estructuraron la comunidad. También destacó que el nivel de disturbio actual del litoral no tuvo efecto sobre la estructura de la comunidad. En adición, el método de segmentación espacial del área de estudio y el diseño de análisis de datos jerarquizado permitió distinguir patrones temporales y espaciales de la densidad, diversidad y composición, y ubicar sitios de alta y baja densidad, y diversidad.

Distribución espacial y temporal de las aves acuáticas en el complejo lagunar
Bahía Magdalena-Almejas: en este estudio se registraron 80 especies de aves acuáticas que se presentaron en diferente temporalidad y se localizaron en diferentes lagunas (ZárateOvando et al. 2006), zonas, habitats y estado de conservación del litoral del complejo lagunar Bahía Magdalena-Almejas. En estudios previos de la costa de isla Santa Margarita (Amador 1985) y el Estero Rancho Bueno (Amador et al. 2006) muy pocos individuos de otras diez especies de aves acuáticas fueron observados.

Durante este estudio, en la costa interna de isla Margarita concurrieron 36 especies en diferentes habitats, 25 en playa arenosa, 15 en costa rocosa, 14 en la costa con vegetación terrestre, 12 en acantilados y 7 en dunas. Y aunque el cormorán orejudo $P h$. auritus tuvo la densidad acumulada mayor, se distribuyó en tan solo 13 de 29 segmentos censados de la costa interna de esta isla pero en siete segmentos tuvo densidad mas alta. En contraste, el pelicano café $P$. occidentalis se distribuyó en 24 de los 29 segmentos y fue la segunda especie con mayor densidad, por lo que su presencia y número de individuos fue mas uniforme a lo largo de la costa insular, seguida de la gaviota occidental $L$. occidentalis. Amador (1985) registró la presencia de 55 especies de aves acuáticas en la costa insular interna, más otras cuatro en la costa externa de la isla. Y similarmente con los resultados de este trabajó encontró que el pelicano café $P$. occidentalis y la gaviota occidental $L$. occidentalis fueron las especies mas frecuentes en las 14 localidades que censó. En este trabajo se registraron áreas de concentración de los cormoranes orejudo $P h$. auritus y de Brandt $P h$. penicillatus en sitios localmente conocidos como Punta Lengua y Las Cabras, mismos que ya se habían identificado anteriormente (Amador 1985). El número menor de especies encontrado en la costa insular interna de isla Margarita en comparación con Amador (1985) se debe en parte, a que en este estudio no se realizaron censos costeros dentro del manglar Las Tijeras, donde Amador registró 12 especies que no se observaron en la línea de costa de la isla en este trabajo, pero de estas Amador (1985) encontró que cinco se distribuyeron solo dentro del manglar (el achichilique 
pico amarillo Aechmophorus occidentalis, la garceta azul Egretta caerulea, la garceta verde Butorides virescens, el pedrete corona negra Nycticorax nycticorax y el chorlo nevado $C h$. alexandrinus), y las otras siete, las registró en otras localidades de la línea de costa interna de la isla, incluyendo el manglar Las Tijeras (la negreta ala blanca Melanita fusca, el pato boludo-menor $A$. affinis, el pato cabeza roja $A$. americana, el mergo copetón Mergus serrator, el mergo mayor $M$. merganser, el zambullidor orejudo Podiceps nigricollis y la garza ganadera Bubulcus ibis), más sin embargo en este estudio, estas especies se registraron en otras áreas (Zárate-Ovando et al. 2006). Por lo anterior, la importancia del manglar Las Tijeras es evidente, ya que con $7.89 \mathrm{~km}$ de perímetro de manglar en planicie de inundación presenta riqueza alta y además mantiene la anidación de la mayor parte de la población de tijeretas de Baja California Sur (Carmona et al. 1995), y colonias de cormoranes y garzas (Amador, 1985) que pudieran ser numéricamente importantes en la península. En total Amador (1985) encontró en Isla Margarita nueve especies (la negreta ala blanca $M$. fusca, el mergo mayor $M$. merganser, la pardela de Revillagigedo $P$. auricularis, el paíño mínimo O. microsoma, el playero solitario T. solitaria, el playero vagabundo $H$. incanus, el playero de Baird C. bairdii, el falaropo pico grueso $P h$. fulicaria y la gaviota blanca L. hyperboreus) que no fueron registradas en el complejo lagunar durante esta investigación (Zárate-Ovando et al. 2006). Análogamente, Amador (1985) no registró seis especies que se observaron en esta investigación, el paíño de Leach Oceanodroma leuchoroa, la garza blanca Ardea alba, el patamarilla mayor Tringa melanoleuca, la gaviota reidora Larus atricilla, el charrán picogrueso Sterna nilotica que además fue nuevo registro para este humedal, y el charrán de Forster S. forsteri.

En el estudio de aves del Estero Rancho Bueno, localidad sureña de este humedal, Amador et al. (2006) observaron entre 43 especies de aves acuáticas, a la espátula rosada (Platalea ajaja) especie que no se observó en nuestros recorridos. En total 90 especies de aves acuáticas se han registrado hasta el momento en el CLBM-A.

En este trabajo, las especies con ubicuidad mayor fueron el pelicano café $P$. occidentalis, el cormorán orejudo $P h$. auritus, la tijereta $F$. magnificens, el gavilán pescador $P$. haliaetus y la gaviota occidental Larus occidentalis ya que estuvieron presentes durante el año, en todas las lagunas, zonas, hábitats y en los tres tipos de estado de conservación del litoral seguidas de otras nueve especies, tales como la garza morena Ardea herodias (salvo en pelágico), el picopando canelo L. fedoa y el chorlo gris Pluvialis squatorola (también ausentes de pelágico y además de acantilados), las gaviotas ploma L. heermannii (excepto en islotes de manglar y planicie de inundación) y de pico anillado L. delawarensis (salvo en islotes de manglar y costa con vegetación terrestre), los charranes caspia Sterna caspia y real S. maxima (ambas especies ausentes de acantilados, islotes de manglar, costa con vegetación terrestre y sustrato antrópico) y los anátidos mas ubicuos fueron el ganso de collar B. bernicla (presente en tres estaciones del año, las tres lagunas y zonas, seis habitats y en segmentos con las tres categorías de conservación) seguida del pato boludo-menor Aythya affinis (en dos estaciones del año, dos lagunas, dos zonas, siete habitats y en las tres categorías de condición).

En esta investigación, a excepción de el pato chillón $B$. clangula que solo se observó en Bahía Magdalena, la mayor riqueza (ZárateOvando et al. 2006) y densidad de anátidos se encontró en Canal Santo Domingo sobre todo en los segmentos norteños que fueron los mas someros de esta laguna, y son sitios de refugio y alimentación. El Canal Santo Domingo es la laguna donde invernan la mayoría de las especies de este grupo y los transectos de esta laguna presentan más de 30 especies, la mayoría segmentos de manglar (ZárateOvando 2007). Es posible que la presencia de alimento en los pastos marinos, la profundidad menor junto con la protección que ocasiona la configuración de los canales, y la estructura vertical del bosque de manglar, sean los factores más importantes que aumenten la riqueza 
de especies y presencia mayor de los anátidos, aves zancudas, buceadoras y playeras en Canal Santo Domingo. Y los hábitos y conducta alimenticia de algunas especies de anátidos y aves buceadoras ocasionan que se distribuyan en sitios con profundidad mayor donde capturan sus presas.

Densidad, diversidad y estructura de la comunidad: el cambio temporal de la densidad y diversidad de primavera-invierno representa patrones cíclicos dirigidos por los procesos migratorios de diferentes especies que afectaron de forma similar a las tres lagunas del área de estudio, pues en invierno y otoño las tres lagunas tuvieron densidad y diversidad significativamente mayor que en primavera, que correspondió con la llegada de aves migratorias tanto norteñas como sureñas. $\mathrm{Y}$ por aumento en el numero de individuos de pelecánidos residentes, que también en esta época realizan movimientos regionales postreproductivos del Golfo de California hacia el complejo lagunar (Anderson y Anderson 1976).

Por otro lado, se puedan hacer comparaciones de la densidad, diversidad o de la similitud en la composición de las aves acuáticas con otros humedales de la península a nivel de laguna o en algunos casos a nivel de habitat. El aumento de la abundancia especifica y riqueza en otoño e invierno, también se ha registrado en humedales norteños como Punta Banda (Escofet et al. 1988), Laguna Ojo de LiebreGuerrero Negro (Carmona y Danemann 1998, Danemann et al. 2002), o en sureños como El Conchalito (Carmona 1995, Carmona y Carmona 2000), Chametla (Fernández-Aceves 1993), el estero de San José del Cabo (Guzmán et al. 1994) o hasta en pequeños embalses de agua residual tratada (Castillo-Guerrero y Carmona 2001), y en todos ellos las parvadas de aves migratorias invernantes elevan considerablemente la abundancia y riqueza de aves acuáticas, como playeros y anátidos. Pero a diferencia de aquellos, las especies más abundantes y de mayor densidad en el complejo lagunar, fueron los pelecánidos (ZárateOvando et al. 2006).
En el área de estudio, la mayoría de las especies fueron poco abundantes (ZárateOvando et al. 2006) o tuvieron densidad baja, lo cual tuvo efecto en los valores de diversidad de los segmentos de las unidades de paisaje o estación del año (Zárate-Ovando 2007).

De los habitats con mayor número de especies, solo el manglar tuvo equidad y diversidad alta, pero en cuanto a la diversidad ésta fue comparable con la diversidad de manglardunas que tuvo $30 \%$ menor riqueza acumulada que manglar, este efecto fue ocasionado por la equidad mayor dentro y entre los segmentos de manglar-dunas debido a que las especies tuvieron densidad mas comparable entre si que en el manglar, donde además se observó el numero mayor de especies "raras" en este estudio. En contraste, de los habitats con menor número de especies destacaron sustrato antrópico y planicie de inundación, en estos habitats se recorrió longitud similar y menor que la recorrida de otros habitats, tuvieron riqueza y diversidad comparable, pero este ultimo indicador fue mucho mayor que el de las dunas y playa arenosa, habitats de mayor cobertura; o que de costa rocosa y manglar-marismas, habitats en los que se recorrió mayor longitud que en aquellos. La dominancia de los pelecánidos en sitios particulares de playa arenosa, dunas y costa rocosa ocasionó este efecto. Y la dominancia de los playeros ocasionó un efecto similar en manglar-marismas.

Las especies del gremio de los pelecánidos que tuvieron densidad acumulada mayor según la estación del año, laguna, zona, habitat y condición de la costa, fueron el cormorán orejudo Ph. Auritus, esta especie tuvo la densidad acumulada mayor desde primavera hasta verano, en las Bahías Magdalena y Almejas, en las costas insular y peninsular, en manglar-dunas y playa arenosa, y en las costas sin disturbio y con disturbio-costa modificada; mientras que el pelicano café $P$. occidentalis mostró la densidad acumulada mayor en acantilados, pelágico, dunas y sustrato antrópico; y el cormorán de Brandt $P h$. penicillatus fue la especie de mayor densidad en otoño en el complejo lagunar. Aunque algunas especies de playeros 
o láridos tuvieron densidad acumulada mas alta que los pelecánidos según la estación del año o unidad de paisaje, el picopando canelo L. fedoa lo fue en invierno, en Canal Santo Domingo en manglar y en planicie de inundación. La dominancia de estas especies causó disminución de los valores de diversidad en los segmentos de habitat donde se concentraron.

En contraste, en humedales de aproximadamente $40 \mathrm{~km}$ de línea de costa interna con menor diversidad de hábitat que la del complejo lagunar a nivel de paisaje, como el estero de Punta Banda, B.C. (con litoral modificado, dunas, marismas y planicie de inundación como hábitats predominantes) Escofet (1988) resaltó la dominancia de especies de playeros sondeadores someros y garzas. En la salina de Guerrero Negro, B.C.S., un área con planicies de inundación y marismas extensas que ocasionan mayor número de microhabitats de gran extensión, los grupos dominantes son los playeros (con el playerito occidental $C$. mauri como especie dominante) seguidos de los anátidos (el ganso de collar B. bernicla y el pato boludo-menor $A$. affinis) y aves buceadoras como el zambullidor orejudo $P$. nigricollis, y en los vasos de cristalización, zona manejada para la producción de sal y rica en Artemia salina, el falaropo de cuello rojo P. lobatus, es la especie dominante. Hacia la región sureña de la península, los playeros como el playerito occidental $C$. mauri también es dominante en la planicie de inundación de La Ensenada de La Paz en sitios donde este habitat es el de mayor cobertura, aunque los pelecánidos (como el pelicano café $P$. occidentalis, el cormorán orejudo $P h$. auritus y la tijereta $F$. magnificens) y láridos (12 especies de gaviotas y siete charranes (Larus spp. y Sterna spp.)) son también conspicuos en playa arenosa, costa rocosa y en el área pelágica de esta región; y en el estero de San José del Cabo (cuerpo de agua salobre, somero, con una área de 40 ha y profundidad promedio de $1.0 \mathrm{~m}$, con tule, carrizo y palmares de palma blanca y datilera como coberturas vegetales principales en su perímetro, los rálidos (la gallereta americana Fulica americana) y anátidos (el pato tepalcate $O$. jamaicensis, y los patos boludo- menor A. affinis y cucharón norteño Anas clypeata) son los grupos dominantes (Guzmán et al. 1994); de manera similar los anátidos es el grupo dominante en embalses de agua tratada (Castillo-Guerrero y Carmona 2001).

La modificación del habitat natural costero y el disturbio ocasionado por el tránsito de embarcaciones aparentemente no tuvieron influencia en los estimadores de la estructura de la comunidad. Sin embargo, la riqueza acumulada menor de aves en el litoral con disturbio-costa modificada (Zárate-Ovando 2007) fue la diferencia principal que indica que este habitat es excluyente. No obstante, el esfuerzo de observación o longitud de litoral recorrido sin disturbio fue mucho mayor que el litoral con disturbio o con costa-modificada. Además en general los pelecánidos y láridos tuvieron densidad alta en sustrato antrópico (incluido el litoral con disturbio-costa modificada) en comparación con los otros tipos de litoral con diferente estado de conservación, lo que sugiere que el efecto del disturbio es más evidente a nivel de gremio o especies dadas sus diferencias de tolerancia y sensibilidad al disturbio.

En conclusión, las diferencias de las condiciones ecológicas del complejo lagunar Bahía Magdalena-Almejas en cuanto a los habitats, sitios de anidación (Zárate-Ovando 2007) y sitios de descanso entre las unidades de paisaje de mayor escala (lagunas y zonas) condicionó la presencia de grupos de aves y afectaron la densidad, diversidad y composición de las especies de la comunidad. La dominancia de pelecánidos muestra que también la condición ecológica marina (Zaytsev et al. 2003, Malakoff 2004, Robinson et al. 2004) es relevante en esta región, tanto que favorece a las aves que se alimentan mayormente de peces y otros recursos marinos, lo que hace necesario considerar que los efectos por reducción de habitat y la sobreexplotación de las pesquerías a futuro dentro del CLBM-A, podrían ocasionar mayor impacto en la comunidad de aves que las fluctuaciones de mesoescala del clima marino provocadas por eventos ENSO, porque aun entre años fríos o cálidos existe alta productividad secundaria dentro del CLBM-A, debido 
a la alternancia en la composición de pelágicos menores (Rodríguez Sánchez et al. 1996).

En adición, aunque en esta investigación la densidad y diversidad de aves acuáticas en el ambiente pelágico fue menor que en costas o habitats, fue en parte por el método de muestreo. En este ambiente dinámico, la densidad y diversidad de aves acuáticas depende de procesos hidrodinámicos que concentran el alimento tales como los frentes de marea, que en temporadas de mayor intensidad en el complejo lagunar ocasionan la concentración masiva de aves y elevan la diversidad en temporadas y zonas específicas como en la Boca Magdalena. Entonces, para la evaluación de los atributos comunitarios de las aves de este ambiente, se deben integrar métodos que consideren las condiciones hidrodinámicas del sistema lagunar.

En cuanto a la especificidad de hábitat, o grado en que las especies fueron generalistas o especialistas en el uso de las unidades de paisaje del CLBM-A fue baja tanto para especies exclusivas de algún hábitat (seis especies concurrieron solo en manglar, y una en dunas, manglar-marismas, playa arenosa o costa rocosa respectivamente; en total $14 \%$ de la comunidad), fueron las especies consideradas como "raras" en este trabajo; como para las especies que usaron solo uno o dos habitats (el manglar fue uno de los dos habitats donde concurrieron seis especies, en la playa arenosa tres especies y la costa rocosa dos especies; es decir, el $28 \%$ de la comunidad de aves uso dos habitats). Esta especificidad de hábitat es baja en comparación con la observada en humedales pequeños como Punta Banda (Escofet 1988), donde el $62 \%$ de las especies desarrolla sus actividades en dos habitats (38\% en uno y $24 \%$ en dos habitats). Esto se explica porque en el área de estudio, 57 especies fueron más generalistas en el uso de habitat, es decir, realizaron sus actividades en más de dos habitats. La especificidad ocasiona que la riqueza regional sea mayor que la local, porque la primera resulta de la suma de las especies exclusivas de cada habitat. En ausencia de especificidad, tanto la riqueza regional como la local son similares. Entonces, en este caso, la comunidad de aves acuáticas del complejo lagunar Bahía Magdalena-Almejas estuvo integrada por mas especies medianamente generalistas al menos de cuatro habitats (manglar 71, dunas 60, playa arenosa 53 y manglar-dunas 51), resultando que la riqueza de estos habitats fuera mas cercana a la riqueza regional (80 especies).

El bosque de manglar del Canal Santo Domingo, el más grande de la península de Baja California Sur, el manglar "Las Tijeras" de isla Margarita y el manglar de Bahía Almejas, albergaron riqueza mayor porque este habitat es sitio de anidación, muda, descanso, refugio y alimentación para las especies dominantes y un mayor espectro de aves acuáticas de diferentes gremios (Zárate-Ovando 2007). Considerando que estos manglares como formación vegetal se localizan en el extremo norteño de la distribución global de los manglares, su importancia queda de manifiesto en el presente trabajo, sin embargo también la playa arenosa y dunas concentraron un espectro amplio de especies. Por lo que, temporalmente estos habitats podrían tener importancia mayor que el manglar comparados a escala menor, porque son sitios de descanso anexos a sitios de alimentación donde regularmente se observan parvadas con densidad y diversidad alta. Con base en los indicadores comunitarios se evidencia la necesidad de implementar acciones de conservación en el manglar, manglar-dunas y segmentos particulares de dunas y playa arenosa.

\section{AGRADECIMIENTOS}

Agradecemos al Acta Norteamericana de Conservación de Humedales (N.A.W.C.A., por sus siglas en ingles) por el financiamiento al proyecto 98210-1G040 que a través de PRONATURA Noroeste A.C. (Dirección de Conservación de B.C.S.), permitió el desarrollo y difusión de esta investigación. A CONACyT y CIBNOR por la beca número 95805. A la colaboración del Centro Pronatura Información para la Conservación del Noroeste de México (CPIC); de Roberto Carmona y equipo de ornitólogos de la Universidad Autónoma de Baja California 
Sur, UABCS; de la Escuela para Estudios de Campo (SFS); y de Rodrigo Rangel.

\section{RESUMEN}

Para probar la hipótesis que la heterogeneidad espacial determina la diversidad de aves acuáticas en un humedal costero, comparamos la densidad, diversidad y composición de especies de aves acuáticas entre habitats y otras unidades de paisaje del complejo Bahía Magdalena-Almejas, Baja California Sur, México. Se realizaron censos de aves acuáticas en 329 segmentos de habitat de la línea de costa interna del complejo lagunar, desde febrero 2002 hasta febrero 2003. La densidad de aves fue mayor en invierno y otoño en las tres lagunas del área de estudio; de las zonas, la densidad fue mayor en la costa insular; y de los habitats en playa arenosa y dunas pero en los pocos segmentos de sustrato antrópico la densidad fue alta. La diversidad fue significativamente mayor en otoño; en Canal Santo Domingo; en la costa peninsular; y de los habitats en manglar-dunas y manglar. La modificación del habitat natural costero y el disturbio por tránsito de embarcaciones no tuvieron influencia en la densidad o diversidad de aves acuáticas, pero la riqueza acumulada mayor ocurrió en las costas bien conservadas y sin disturbio. El análisis de la composición de especies mostró diferencias entre las asociaciones de Canal Santo Domingo y Bahía Magdalena. El ambiente pelágico y el manglar tuvieron diferencia mayor en la composición de especies con respecto a las asociaciones de los demás habitats, tales diferencias en elencos de especies por habitat sugieren que la comunidad de aves está estructurada de acuerdo a los recursos naturales disponibles de las lagunas, zonas y habitats, en relación con la complejidad estructural de las unidades de paisaje. Las condiciones ecológicas del complejo lagunar ocasionan que las aves acuáticas piscívoras sean el grupo dominante de esta comunidad.

Palabras clave: aves acuáticas, densidad, estructura de la comunidad, análisis espacial, Bahía Magdalena-Almejas, B.C.S.

\section{REFERENCIAS}

Amador, S.E. 1985. Avifauna de isla Santa Margarita, Baja California Sur, México. Tesis de licenciatura, Univ. Autón. de Baja California Sur, La Paz, México.

Amador, S.E., R.A. Mendoza-Salgado \& J.A. de AndaMontañez. 2006. Estructura de la avifauna durante el periodo invierno-primavera en el Estero Rancho Bueno, Baja California Sur. Rev. Mex. Biodivers. 77: 251-259.
Anderson, D.W. \& I.T. Anderson. 1976. Distribution and status of Brown Pelicans in the California Current. Am. Birds 30: 3-12.

Becker, B.H. \& S.R. Beissinger. 2003. Scale-dependent habitat selection by nearshore seabird, the marbled murrelet, in a highly dynamic upwelling system. Mar. Ecol. Prog. Ser. 256: 246-255.

Butler, R.W., R.I.G. Morrison, F.S. Delgado, R.K. Ross \& G.E.J. Smith. 1997. Habitat associations of coastal birds in Panama. Colon. Waterbirds 20: 518-524.

Carmona, R. 1995. Distribución temporal de aves acuáticas en la playa El Conchalito, Ensenada de La Paz, Baja California Sur. Inv. Mar. CICIMAR 10: 1-25.

Carmona, R. \& C. Carmona. 2000. Abundancia y riqueza específica de aves playeras en la playa "El Conchalito" Baja California Sur, durante 1993 y 1995. Hidrobiológica 10: 69-78.

Carmona, R. \& G. Danemann. 1998. Distribución espaciotemporal de aves en la salina de Guerrero Negro, Baja California Sur, México. Cienc. Mar. 24: 389-408.

Carmona, R. \& G.D. Danemann. 2000. Distribución espacio-temporal de aves Pelecaniformes en la salina de Guerrero Negro, Baja California Sur, México. Hidrobiológica 10: 85-90.

Castillo-Guerrero, J.A. \& R. Carmona. 2001. Distribución de aves acuáticas y rapaces en un embalse dulceacuícola artificial de Baja California Sur, México. Rev. Biol. Trop. 49: 1131-1142.

Clarke, K.R. \& R.M. Warwick. 2001. Change in marine communities: an approach to statistical analysis and interpretation. Primer-E LTD, Plymouth, Reino Unido.

Danemann, G.D., R. Carmona \& G. Fernández. 2002. Migratory shorebirds in the Guerrero Negro Saltworks, Baja California Sur, Mexico. Wader Study Group Bulletin 97: 36-41.

Escofet, A., D.H. Loya-Salinas \& J.I. Arredondo. 1988. El estero de Punta Banda (Baja California, México) como habitat de la avifauna. Cienc. Mar. 14: 73-100.

Fernández-Aceves, G.J. 1993. Importancia de la marisma de Chametla, Ensenada de La Paz, B.C.S., para la migración e invernación del Playerito Occidental (Calidris mauri) (Charadriiformes: Scolopacidae). Tesis de licenciatura, Universidad Autónoma de Baja California Sur, La Paz, México. 
Fernández, G., R. Carmona \& H. De la Cueva. 1998. Abundance and seasonal variation of western sandpipers (Calidris mauri) in Baja California Sur, México. Southwest. Nat. 43: 57-61.

Erickson, R.A. \& S.N.G. Howell. 2001. Birds of the Baja California Peninsula: Status, Distribution, and Taxonomy. Monographs in Field Ornithol. 3. Amer. Birding Assoc., Colorado Springs, Colorado, EEUU.

Grinnell, J. 1928. A Distributional Summation of the Ornithology of Lower California. Univ. Calif. Publ. Zool. 32: 1-300.

Guzmán, J., R. Carmona, E. Palacios \& M. Bojórquez. 1994. Distribución temporal de aves acuáticas en el estero de San José del Cabo, Baja California Sur, México. Cienc. Mar. 20: 93-103.

Hattori, A. \& S. Mae. 2001. Habitat use and diversity of waterbirds in a coastal lagoon around Lake Biwa, Japan. Ecol. Res. 16: 543-553.

Howell, S.N.G. 2001. Regional Distribution of the Breeding Avifauna of the Baja California Peninsula, p. 10-22. In R.A. Erickson \& Howell S.N.G. (eds.). Birds of the Baja California Peninsula: Status, Distribution, and Taxonomy. Monographs in Field Ornithology 3 . Amer. Birding Assoc., Colorado Springs, Colorado, EEUU.

Howell, S.N.G., R.A. Erickson, R.A. Hamilton \& M.A. Patten. 2001. An Annotated Checklist of the Birds of Baja California and Baja California Sur, p 171-203. In R.A. Erickson \& S.N.G. Howell (eds.). Birds of the Baja California Peninsula: Status, Distribution, and Taxonomy. Monographs in Field Ornithology 3. Amer. Birding Assoc.,Colorado Springs, Colorado, EEUU.

Jaquement, S., M. Le Corre \& H. Weimerskirch. 2004. Seabirds community estructure in a coastal tropical envirronment: importance of natural factors and fish aggregating devices (FADs). Mar. Ecol. Prog. Ser. 268: 281-292.

Kasprzyk, M.J. \& B.A. Harrington. 1989. Manual de campo para el estudio de aves playeras. Playeras RHRPA, editor. Ensenada, Baja California. CICESE MBO, Ensenada, Mexico.

Malakoff, D. 2004. New tools reveals treasures at ocean Hot Spots. Science 304: 1104-1105.
Massey, B.W. \& E. Palacios. 1994. Avifauna of the wetlands of Baja California, México: current status, p 45-47. In J.R. Jehl, Jr. \& N.K. Johnson (eds.). Studies of Avian Biology 15: A Century of Avifaunal Change in Western North America. Cooper Ornithol. Society, Lawrence, Kansas, EEUU.

National Geographic Society. 2002. Field Guide to the Birds of North America. NG Society, Washington D.C., EEUU.

Nelson, E. 1920. Lower California and its Natural Resources. Natl. Acad. of Sciences, Washington, DC, EEUU.

Page, G.W., E. Palacios, L. Alfaro, S. González, L.E. Stenzel \& M. Jungers. 1997. Numbers of wintering shorebirds in coastal wetlands of Baja California, Mexico. J. Field Ornithol. 68: 562-574.

Palacios, E., L. Alfaro \& G.W. Page. 1994. Distribution and abundance of breeding snowy plovers on the Pacific coast of Baja California. J. Field Ornithol. 65: 490-497.

Robinson, C.J., V. Anislado \& A. López. 2004. The pelagic red crab (Pleuroncodes planipes) related to active upwelling sites in the California Current off the west coast of Baja California. Deep-Sea Research II 51: 753-766.

Rodríguez Sánchez, R., S. Hernández-Vázquez, D. LluchBelda, R. Félix-Uraga, S. Ortega, A. Villa Arce, G. Ponce-Díaz \& D.B. Lluch-Cota. 1996. Pesquerías de Pelágicos Menores (Sardinas y Anchovetas). In M. Casas Valdez \& G. Ponce-Díaz (eds.). Estudio del Potencial Pesquero y Acuícola de Baja California Sur. B.C.S. Secretaria de Medio Ambiente y Recursos Naturales y Pesca, La Paz, Baja California Sur, México.

Roshier, D.A., A.I. Robertson \& R.T. Kingsford. 2001. Continental-scale interactions with temporary resources may explain the paradox of large populations of desert waterbirds in Australia. Landscape Ecol. 16: 547-556.

Saad, N.G. \& E. Palacios. 2004. Planificación para la Conservación de Sitios: Complejo Lagunar de Bahía Magdalena-Almejas, B.C.S., México. Pronatura A.C. (Noroeste), La Paz, Baja California Sur, México.

Sibley, D.A. 2001. National Audobon Society. The Sibley Guide to Birds. Alfred A. Knopf, Nueva York, EEUU. 
Weller, M.W. 1999. Wetland birds. Habitat resources and conservations implications. Cambridge, Cambridge, Inglaterra.

Wilbur, S.R. 1987. Birds of Baja California. Berkeley: Univ. of Calif., California, EEUU.

Zárate-Ovando, B., E. Palacios, H. Reyes-Bonilla, E. Amador \& G. Saad. 2006. Waterbirds of the Lagoon Complex Magdalena Bay-Almejas, Baja California Sur, México. Waterbirds 29: 350-364.
Zárate-Ovando, B. 2007. Ecología y Conservación de las aves acuáticas del complejo lagunar Bahía Magdalena-Almejas, B.C.S., México. Tesis de Doctorado, Centro de Investigaciones Biológicas del Noroeste, La Paz, Baja California Sur, México.

Zaytsev, O., R. Cervantes-Duarte, O. Montante \& A. Gallegos-García. 2003. Coastal upwelling activity on the Pacific shelf of the Baja California peninsula. J. Oceanog. 59: 489-502. 
